\title{
Computed tomography lymphangiography via intrametatarsal pad injection is feasible in cats with chylothorax
}

Chuan Chiang, DVM¹; Kuan-Sheng Chen, PhD, MANZCVS, DVM²,3; Hsien-Chieh Chiu, MS, DVM4; Cheng-Shu Chung, PhD, DVM5,7; Lee-Shuan Lin, PhD, DVM6,7*

\begin{abstract}
${ }^{1}$ UniCore Animal Hospital, Taipei City, Taiwan
2Department of Veterinary Medicine, College of Veterinary Medicine, National Chung Hsing University, Taichung, Taiwan

3 Veterinary Medical Teaching Hospital, College of Veterinary Medicine, National Chung Hsing University, Taichung, Taiwan

${ }^{4}$ Tzuoo Ann Animal Hospital, New Taipei City, Taiwan

${ }^{5}$ Laboratory of Veterinary Surgery, Department of Veterinary Medicine, College of Veterinary Medicine, National Pingtung University of Science and Technology, Pingtung, Taiwan

${ }^{6}$ Laboratory of Veterinary Diagnostic Imaging, Department of Veterinary Medicine, College of Veterinary Medicine, National Pingtung University of Science and Technology, Pingtung, Taiwan

${ }^{7}$ Veterinary Medical Teaching Hospital, College of Veterinary Medicine, National Pingtung University of Science and Technology, Pingtung, Taiwan
\end{abstract}

*Corresponding author: Dr. Lin (linleeshuan@gmail.com)

https://doi.org/10.2460/ajvr.21.10.0163

\section{OBJECTIVE}

To evaluate the feasibility of CT Iymphangiography via intrametatarsal pad injection in cats with chylothorax.

\section{ANIMALS \\ 7 client-owned cats.}

\section{PROCEDURES}

This was a multicenter, retrospective, descriptive study. Medical records and imaging data from 4 veterinary hospitals were reviewed to identify cats with chylothorax that had undergone intrametatarsal pad injection via CT lymphangiography. In total, 7 client-owned cats were included in the study. Signalment, history, image findings, and follow-up data were recorded. Descriptive statistics were used to analyze the success rate of thoracic duct (TD) enhancement and describe relevant clinical findings.

\section{RESULTS}

Enhancement of TDs was successful in 6 of the 7 cats within 5 to 15 minutes after initiating intrametatarsal pad injection under general anesthesia. Successful migration of contrast medium into the lymphatic vessels cranial to the popliteal lymph nodes was observed in all cats within 5 minutes after injection. The recommended dose of contrast medium to achieve TD enhancement was $1 \mathrm{~mL} / \mathrm{kg}(0.5 \mathrm{~mL} / \mathrm{kg} / \mathrm{pad}$; concentration, $350 \mathrm{mg}$ of iodine/ kg). Only 1 cat had mild swelling of the paws after the procedure, and it recovered quickly without pain medication; no cats experienced lameness. Similar to dogs and unlike in previously published reports, 72\% of TD branches were located in the right hemithorax.

\section{CLINICAL RELEVANCE}

CT Iymphangiography via intrametatarsal pad injection is a feasible and safe procedure for cats with chylothorax. This technique provides detailed information regarding the unique TD anatomy and cisterna chyli location. It also contributes to surgical planning.

n cats, chylothorax is caused by an abnormal flow or pressure in the thoracic duct (TD) and its branches, leading to chylous accumulation in the thoracic cavity. ${ }^{1}$ Multiple possible etiologies, including cardiomyopathy, neoplasia, congenital cardiac abnormalities, thrombosis, lung lobe torsion, and dirofilariasis, have been determined in feline chylothorax, but most cases are considered idiopathic. ${ }^{2-9}$ Surgical intervention is necessary if medical management is ineffective or impractical. ${ }^{1}$ Surgical intervention is warranted in animals with idiopathic chylothorax or those that are not responsive to medical management. ${ }^{1}$ Among surgical options, TD ligation, subtotal pericardiectomy, cisterna chyli ablation, thoracic embolization, and thoracic omentalization are commonly considered effective. 5,10-13 In cats, a combination of TD ligation with subtotal pericardiectomy has a higher success rate (73\%) for clinical resolution than performing TD ligation alone (41\%). ${ }^{14,15}$ However, unlike the improved success rate when cisterna chyli ablation is combined with TD ligation in dogs (83\%), 1 study ${ }^{14}$ indicated that the strategy did not result in better outcomes in cats. 
Owing to the high variation of Iymphatic vessels among individuals, identifying their position and branches during TD ligation is important. Intraoperative lymphangiography is commonly performed by injection of methylene blue or indocyanine green via mesenteric lymph node injection during laparotomy or feeding cream or corn oil before induction. 5,10,16 Preoperative CT lymphangiography is helpful for surgical planning and determining the approach site. Ultrasound-guided injection of contrast medium into the mesenteric or popliteal lymph nodes in chylothorax cases has been reported. ${ }^{17-22}$ Nevertheless, these techniques have failed in some feline cases because of the difficulty of injecting large amounts of contrast medium into the small lymph nodes of cats. ${ }^{17,22}$

Simpler alternative methods for CT lymphangiography by SC injection of the contrast medium into perianal tissue or the dorsal metatarsal region have been implemented in dogs. However, it may be difficult to inject contrast medium into the small perianal region of cats, and similar to dogs, infection is also a concern. ${ }^{23-26}$ Moreover, the potential needle trauma to the dorsal tendons and adjacent nerves during the injection because of the thin subcutis at the dorsal metatarsal region, particularly in small-sized animals, cannot be ignored. We previously reported the feasibility of CT lymphangiography via intrametatarsal pad injection as a safe and straightforward method for dogs. ${ }^{20}$ In this method, the thick subcutaneous adipose tissue of the pad allows for the injection of large amounts of contrast medium, enabling satisfactory visualization of lymphatic vessels, cisterna chyli, and the TD.

This retrospective study aimed to assess the outcomes of CT lymphangiography via intrametatarsal pad injection in cats with chylothorax and to describe related clinical findings.

\section{Materials and Methods}

\section{Case selection}

This was a multicenter, retrospective, descriptive study. We reviewed medical records and imaging data obtained between July 1, 2018, and November 30, 2020, from the Veterinary Medical Teaching Hospital of National Pingtung University of Science and Technology (Pingtung, Taiwan), National Chung Hsing University Veterinary Medical Teaching Hospital (Taichung City, Taiwan), Tzuoo Ann Animal Hospital (New Taipei City, Taiwan), and UniCore Animal Hospital (Taipei City, Taiwan). Each hospital approved data usage. The inclusion criteria were client-owned cats with chylothorax that underwent CT Iymphangiography via intrametatarsal pad injection with nonionic iodinated contrast medium. Patient data were excluded if they previously had TD surgery. This work involved the use of nonexperimental animals alone.

\section{Imaging equipment}

Four helical multidetector CT scanners with different imaging techniques were used. Two 16-slice multidetector CTs and two 64-slice multidetector
CTs were used. The CT parameter details are listed elsewhere (Supplementary Appendix S1).

\section{CT scan and intrametatarsal pad lymphangiography}

Animals were anesthetized and maintained on gas anesthetic and subsequently positioned in sternal recumbency for CT scan. After location acquisition, helical precontrast scanning was performed.

Lymphangiography was performed after aseptic preparation of the pads. ${ }^{20}$ The cats were injected with a nonionic iodinated contrast medium (iohexol [Omnipaque 350], $350 \mathrm{mg}$ of iodine/kg; or ioversol [Optiray 350], $350 \mathrm{mg}$ of iodine $/ \mathrm{kg}$ ) into the right and left hind limb metatarsal pads in equal aliquots with 21- or 22-gauge needles for 15 to 30 seconds. The metatarsal pads, distal hind limbs, and tarsal and thigh regions were massaged thoroughly from the distal to proximal direction for 3 to 4 minutes after the injection (Figure 1; Supplementary Video V1). The CT scan was performed 5 minutes after the intrametatarsal pad injection. If the contrast medium did not opacify the TD within 5 minutes of lymphangiography, the scans were repeated at 2- to 3-minute intervals until satisfactory TD enhancement was obtained. Postcontrast IV contrast CT scan was performed after lymphangiography with a dose of 2 $\mathrm{mL} / \mathrm{kg}$.

\section{Data recording}

Clinical data-Signalment (sex, breed, and body weight), CT imaging parameters, lymphangiography techniques used (contrast medium, injected volume, injection method, and intervals of delayed scans), adverse effects, and follow-up information were summarized from the medical records for each cat by 1 veterinarian (CC).

Imaging analysis-The $\mathrm{CT}$ images were reviewed in the soft tissue window (window level, $40 \mathrm{HU}$; window width, $400 \mathrm{HU}$ ) with commercial software (Osirix MD version 11.0.3; Pixmeo SARL). Qualitative and quantitative data were evaluated by 2 authors (CC and LSL). Observers could see the patient identification number and name but were unaware of the signalment, injected contrast volume, injection method, and outcome at the time of evaluation. The 3-D images were processed using a commercial imaging workstation (Attractive; PixSpace Ltd).

\section{Identification of lymphatic vessels and evaluation of TD enhancement}

Successful lymphangiography was defined as lymphatic vessel enhancement cranial to the popliteal lymph nodes. Enhancement of TD was deemed successful when the contrast medium enabled its identification. The number of visible TD branches was evaluated and recorded at each midvertebral region from T9 to L1. ${ }^{20,21}$ At the common TD ligation site at the T10 to T12 level, location of the TD branch relative to the aorta was expressed using a clockface analogy. The quality of TD enhancement at the T10 to T12 level was graded as follows: grade 3 
(excellent), grade 2 (good), grade 1 (fair), and grade 0 (poor). ${ }^{20}$ The distance between the TD and aorta was assessed. A line was drawn from the center of the aorta to the TD branches at the midvertebral region from T10 to T12, and the distance from the aortic wall to the TD branches was measured.

\section{Evaluation of the cisterna chyli}

The location of the cisterna chyli relative to the aorta and the pattern of the structure were assessed and recorded. The length of the cisterna chyli was also measured on sagittal planes.

\section{Evaluation of the efferent lymphatic pathway}

If the hind limbs and pelvic area were included in the scan, the pattern of the lymphatic efferent pathway was evaluated.

\section{Data analysis}

Statistical analyses were performed with a spreadsheet (Excel for Mac version 16.45; Microsoft Corp) and commercial software (Prism version 8.0; GraphPad Software Inc). A preliminary Shapiro-Wilk test was performed, and it demonstrated non-normally distributed data. Age and body weight were expressed as median and range values.

\section{Results}

\section{Study population}

Seven cats met the inclusion criteria and included 2 Ragdoll and 5 mixed-breed cats. The median
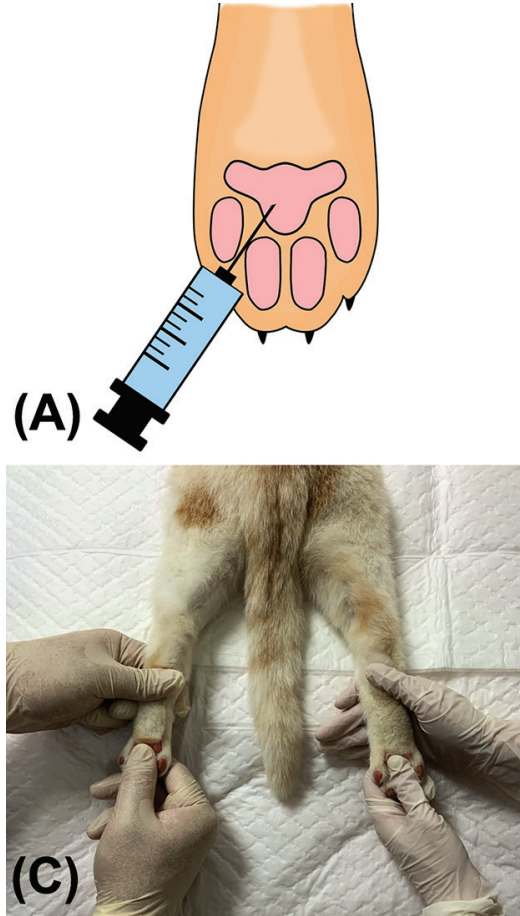

Figure 1-Intrametatarsal pad CT Iymphangiography in a cat. Illustration (A) and photograph (B) of intrametatarsal pad injection of the contrast medium. The pads (C) and hind limbs (D) were massaged for 3 to 4 minutes after injection. age was 7 years (range, 2 to 15 years) and median body weight was $4.2 \mathrm{~kg}$ (range, 3.2 to $6.8 \mathrm{~kg}$ ); 4 cats were spayed females, 2 were neutered males, and 1 was a sexually intact male.

\section{CT scan and intrametatarsal pad lymphangiography}

Prior to general anesthesia, thoracocentesis was performed in 5 cats, and the volume of aspirated chylous effusion ranged from 100 to $150 \mathrm{~mL}$. The CT scanning included the thoracic, abdominal, and pelvic limb regions in 6 cats; 1 cat was only scanned from the thoracic inlet to the L4 level. One cat showed no TD enhancement even after repeated scans of lymphangiography. Therefore, 30 minutes after intrametatarsal pad injection, the scanning was discontinued.

The median dose of contrast medium for lymphangiography was $1.25 \mathrm{~mL} / \mathrm{kg}$ (range, 1.0 to 2.0 $\mathrm{mL} / \mathrm{kg}$ ). The doses used in the 6 cases of successful TD enhancement were as follows: $1 \mathrm{~mL} / \mathrm{kg}$ in 3 cats, $1.5 \mathrm{~mL} / \mathrm{kg}$ in 1 cat, and $2 \mathrm{~mL} / \mathrm{kg}$ in 2 cats. One cat was administered contrast medium at a dose of 1.5 $\mathrm{mL} / \mathrm{kg}$, but no TD enhancement occurred.

\section{Timing of lymphatic vessel and TD enhancement}

For all cats, Iymphatic enhancement could be visualized cranial to the popliteal lymph node within 5 minutes after injection, indicating a 100\% success rate of lymphangiography via intrametatarsal pad injection.

Enhancement of TD was identified in 6 of 7 cats within 15 minutes after injection. The TD was successfully identified within 5 minutes in 1 cat (14\%) in the first scan and within 6 to 15 minutes by repeated scanning in 5 cats. No TD enhancement was found in 1 cat, in which the contrast medium accumulated in bilateral enlarged medial iliac lymph nodes.

\section{Quality and quantity of TD enhancement}

The quality of the TD enhancement at the T10 to T12 level was as follows: 4 cats exhibited grade 3 enhancement, 2 exhibited grade 2 enhancement, and 1 exhibited grade 0 enhancement. A fused 3-D image from the lymphangiography and angiography was created (Figure 2).

Multiple TD branches were identified in 6 cats with successful TD enhancement. The maximum numbers of TD branches were as follows: 2 in 3 cats, and 3 in the other 3 cats. The number of TD branches at different vertebral levels was variable (Supplementary Table S1).

A clockface analogy of the TD branches from a craniocaudal view at 

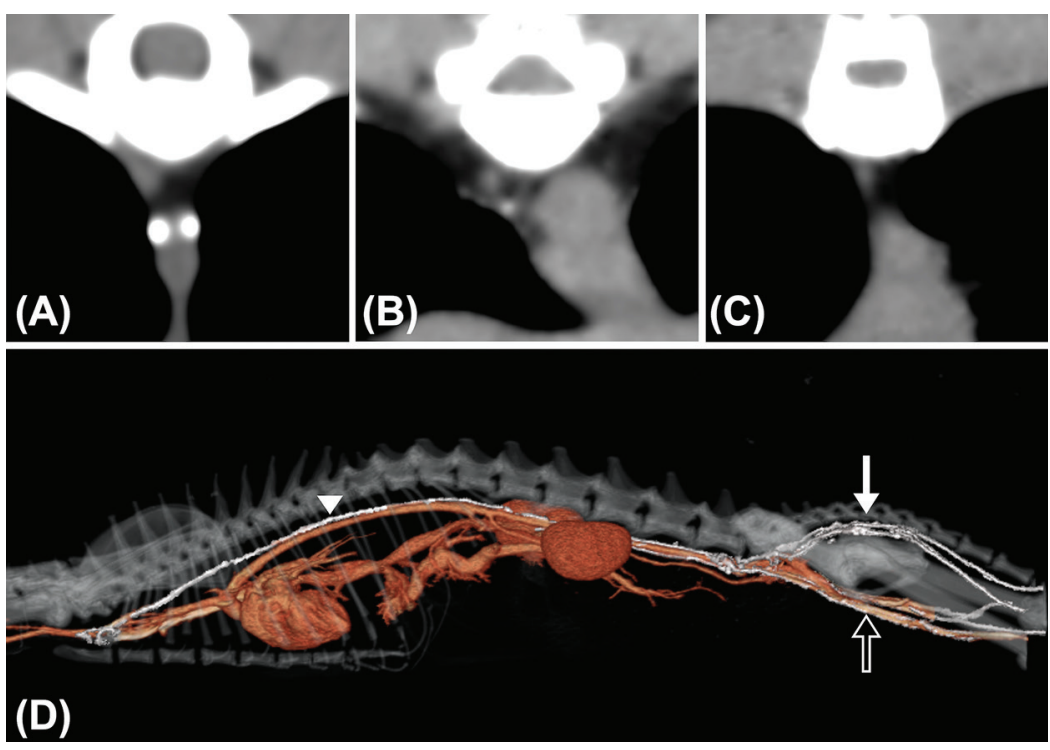

Figure 2-Grading and percentage of thoracic duct (TD) enhancement at the T10 to T12 level. A-Grade 3 (excellent). All branches are intensively enhanced ( $4 / 7$ cats in this study). B-Grade 2 (good). The main branch is intensively enhanced with suboptimal enhancement of smaller branches (2 cats). C-Grade 0 (poor; 1 cat). No enhancement. No cats showed grade 1 (fair). Panels $A$ through $C$ are transverse $C T$ images with the following parameters: window level, $40 \mathrm{HU}$; window width, $400 \mathrm{HU}$; soft tissue algorithm, 0.625- to 1-mm slice thickness. D-Representative 3-D image of a cat with chylothorax. This is a fused image of Iymphangiography (white) and CT angiography (red). The lymph vessels from the popliteal to the pelvic region, including the ventral (white-outlined arrow) and dorsal lymph vessel (white arrow) pathways, are seen, and the TD (arrowhead) is well visualized.

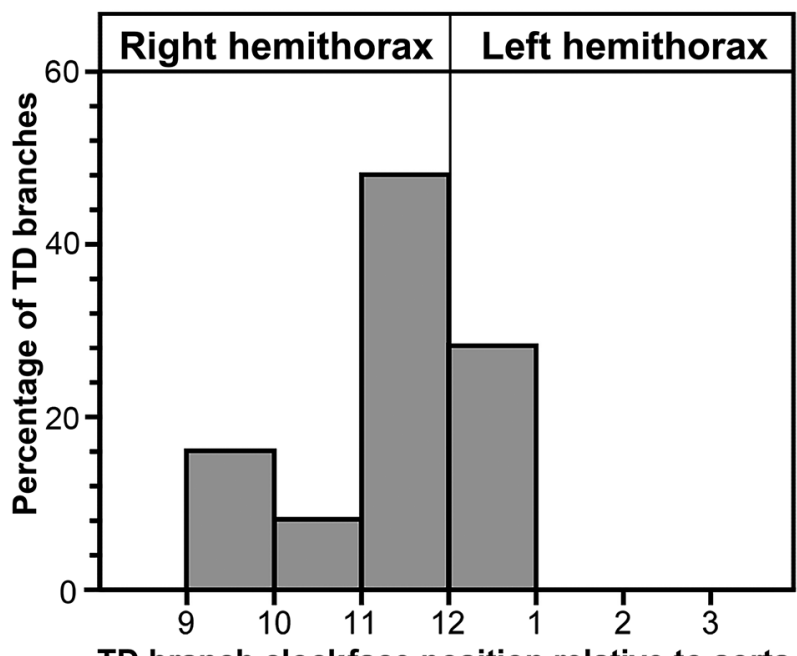

TD branch clockface position relative to aorta

Figure 3-Graph of the TD branch location relative to the aorta at the T10 to T12 vertebral segment using a clockface analogy from the craniocaudal view of the CT images for the cats in this study.

the T10 to T12 vertebral level was determined (Figure 3). Seventy-two percent of TD branches were located in the right hemithorax, between the 9:00 and 12:00 positions relative to the aorta, whereas $28 \%$ were found in the left hemithorax between the 12:00 and 1:00 positions. Leakage of contrast medium into the mediastinum was not identified in any of the cases.
The distance between the TD and the aorta was measured at the T10 to T12 level. In almost all cats with successful TD enhancement (5/6 cats), the TDs were located adjacent to the aorta or less than $1 \mathrm{~mm}$ from it, except for 1 cat with a distance of 2.6 to $3.4 \mathrm{~mm}$ from the aorta at the T10 to T12 level.

\section{Cisterna chyli location and structure}

Successful cisterna chyli enhancement was noted in 6 cats, and the location ranged from $L 2$ to $L 3$. Five different types of cisterna chyli structures were identified, as follows: right to the aorta ( $n=1$ cat); dorsal (1); right and dorsal (1); right, dorsal, and ventral (2); and spiral (1; Figure 4). The median length of the cisterna chyli was $1.25 \mathrm{~cm}$ with a range of 1.0 to $3.3 \mathrm{~cm}$.

\section{Lymphatic pathway assessment}

The hind limb and pelvic regions were included in the scanning field in 6 cats. This allowed for evaluation of the direct Iymphatic efferent pathway from the popliteal lymph nodes to the medial iliac lymph nodes and the dorsal lymph vessel pathway coursing to the iliac Iymph nodesalong the gluteal region. ${ }^{27}$ Bilateral direct efferent pathways were observed in all 6 cats. Four cats had dorsal lymph vessel pathways as follows: 1 exhibited a bilateral dorsal lymph vessel pathway, whereas 3 exhibited right dorsal lymph vessel pathways (Figure 5).

\section{Follow-up}

Changes in appetite and abnormal clinical signs, such as pain, lameness, or swelling of the paws, were determined from the medical reports or with follow-up calls. Only 1 cat had mild swelling of the paws after the procedure, and it recovered the next day. No further side effects were observed during the procedure.

Three cats had been speculated to have concurrent diseases. Cat 1 was diagnosed with right cardiomegaly and pulmonary hypertension, and chylothorax gradually resolved within 2 months after administration of pimobendan (Vetmedin) and sildenafil (Viagra). Cat 6 had multiple large abdominal masses with concurrent chylothorax and peritoneal effusion. Granulomatous disease as in feline infectious peritonitis or lymphoma was suspected by means of cytologic evaluation of the masses, and coronavirus was detected in the pleural fluid; the cat's clinical condition deteriorated, and it died 2 weeks after the CT examination. The TD enhancement failed in cat 7 . In this cat, a mass at the level of the right third mammary gland and extremely enlarged ipsilateral inguinal and bilateral medial iliac lymph nodes were noted on the CT images; thus, 


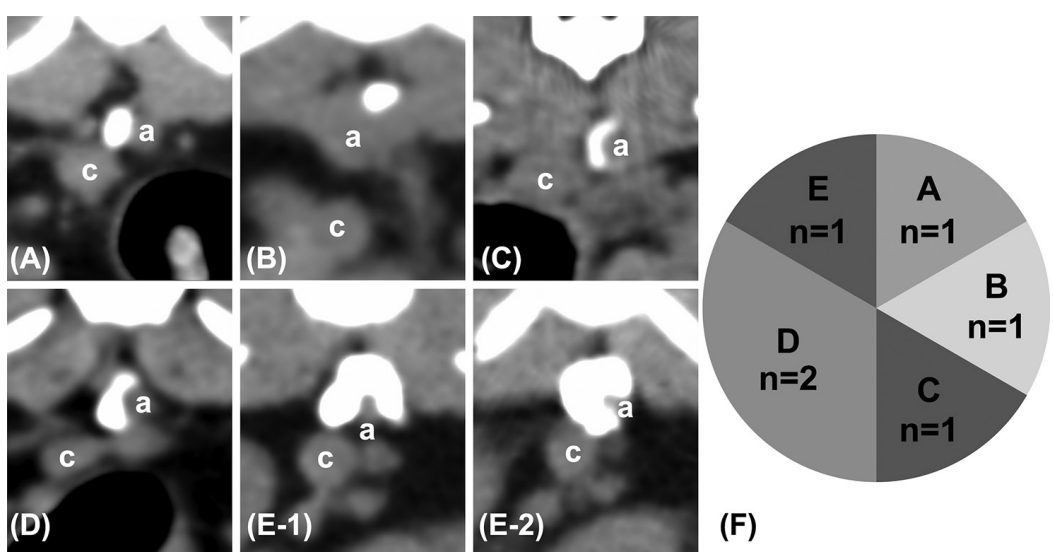

Figure 4-Computed tomographic images (panels A through E) of variable cisterna chyli structure among individual cats. Different structural types of the cisterna chyli are seen with respect to the aorta as follows: right to the aorta (A); dorsal (B); right and dorsal (C); dorsal, right, and ventral (D); and spiral (E-1 and E-2). The cisterna chyli is right, dorsal, and left to the aorta in the cranial portion (E-1) and then courses to a position ventral, right, and dorsal to the aorta in the caudal portion (E-2). F-Chart of the prevalence of each type among the 6 cats with enhanced cisterna chyli. $\mathrm{a}=$ Aorta. $\mathrm{c}=$ Caudal vena cava. See Figure 2 for CT parameters.
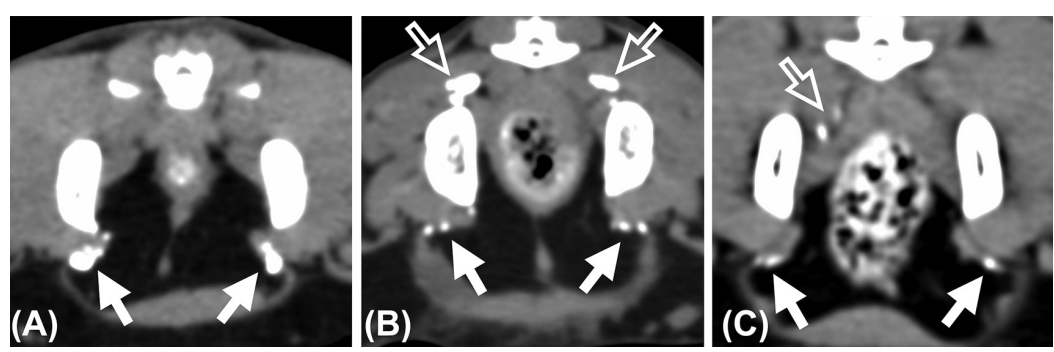

Figure 5-Computed tomographic images of variable lymphatic drainage pathways of the hind limb. The white arrows indicate lymphatic vessels directly draining from the popliteal lymph nodes to the medial iliac lymph nodes along the medial proximal hind limb. In contrast, the white-outlined arrows indicate dorsal lymph vessel pathways from the dorsal gluteal regions. The cats exhibited a bilateral medial pathway $(A)$, bilateral medial and dorsal lymph vessel pathway (B), or unilateral dorsal lymph vessel pathway (C). See Figure 2 for CT parameters.

mammary gland tumor with Iymphatic metastasis was speculated. However, the owner declined further biopsy, and the cat was lost to follow-up after the CT examination and no definitive diagnosis could be made.

Four cats were diagnosed with idiopathic chylothorax, and 3 cats underwent video-assisted thoracoscopic surgery as follows: TD ligation in 2 cats (cats 2 and 3), and TD ligation with subtotal pericardiectomy in 1 cat (cat 4 ). These 3 cats exhibited right-sided distribution of TDs on preoperative CT lymphangiography; thus, a right-sided approach for TD ligation was selected. An intrametatarsal pad injection of indocyanine green was performed for intraoperative lymphangiography in 2 cats (cats 3 and 4), and both showed concordant identification of the TD branches with the CT lymphangiographic findings. Thoracic tubes were placed in all 3 cats for monitoring postoperative pleural effusion, and all cats had clear effusion after surgery and tapped from 33 to $100 \mathrm{~mL} / \mathrm{d}$ to $<3 \mathrm{~mL} / \mathrm{d}$ in 1 to 2 weeks. There was no recurrence in the cases treated with surgery after 3 to 9 months of followup. Cat 5 with idiopathic chylothorax was lost to follow-up after the CT examination. The details of each cat are listed elsewhere (Supplementary Appendix S2).

\section{Discussion}

The present study demonstrated CT lymphangiography via intrametatarsal pad injection in cats with chylothorax as a simple procedure with a high success rate (6 of 7 cats) within 5 to 15 minutes of injection. This approach allows for identification of lymphatic vessels, cisterna chyli location, and the variable TD branch locations. Moreover, successful migration of the contrast medium from the injection site to the lymphatic vessels occurred in all cases within 5 minutes. The recommended procedure is similar to that in a previous report 20 in dogs, as follows: prepare at least $1 \mathrm{~mL}$ of contrast medium $/ \mathrm{kg}(0.5 \mathrm{~mL} / \mathrm{kg} / \mathrm{pad})$ and inject into the thickest region of the metatarsal pads at the same time; thoroughly and vigorously massage the pads and hind limbs for 3 to 4 minutes, then start the first CT scan within 5 minutes after initiating the injection. Failure to opacify the TD by the first scan means that serial delayed scans at 2- to 3-minute intervals may be necessary until a satisfactory TD enhancement is achieved.

The present study demonstrated a high success rate $(86 \%)$ of CT lymphangiography via intrametatarsal injection in cats, comparable with the success rate in dogs (90\%). ${ }^{20}$ Previous reports have demonstrated differing success rates of ultrasoundguided intranodal (popliteal or mesenteric lymph nodes) injection for CT lymphangiography in cats ( $71 \%$ to $100 \%$ ). Difficulty in the injection of the contrast medium into the popliteal lymph nodes and extranodal contrast leakage in the abdominal cavity via intramesenteric lymph node injection have also been described. ${ }^{17,22,28}$ In comparison, SC injection of contrast medium is less technically demanding and shows no abdominal leakage, either from the perianal region, dorsal metatarsal region, or intrametatarsal pads. ${ }^{20,23-25}$

Thoracic duct enhancement may be influenced by the volume of the contrast medium used. ${ }^{28,29}$ Previous studies in dogs suggest a dose of $1 \mathrm{~mL}$ of contrast medium $/ \mathrm{kg}$ by popliteal lymph node injection and 0.75 to $1 \mathrm{~mL} / \mathrm{kg}$ by metatarsal SC injection, whereas a lower dose (1.5 mL/cat) has been recommended in cats for popliteal lymph node injection or mesenteric lymph node injection. ${ }^{17,21,22,25,28}$ The 
most frequently used dose of contrast medium in our study was $1 \mathrm{~mL} / \mathrm{kg}$, similar to the dose used for intrametatarsal injection in dogs. ${ }^{20}$ Among the 6 cats with successful TD enhancement in the present study, grade 3 enhancement (4/6 cases) was achieved with contrast medium doses of $1 \mathrm{~mL} / \mathrm{kg}(\mathrm{n}=2), 1.5 \mathrm{~mL} /$ $\mathrm{kg}(1)$, and $2 \mathrm{~mL} / \mathrm{kg}(1)$, whereas grade 2 enhancement ( $2 / 6$ cases) was achieved with a contrast medium dose of $1 \mathrm{~mL} / \mathrm{kg}$ (1) and $2 \mathrm{~mL} / \mathrm{kg}$ (1). One cat received an injection of $1.5 \mathrm{~mL}$ of contrast medium/ $\mathrm{kg}$, but no TD enhancement was achieved. A higher dose of contrast medium did not guarantee better quality of TD visualization, similar to the findings of a previous report 20 in dogs. Thus, an initial dose of $1 \mathrm{~mL}$ of contrast medium $/ \mathrm{kg}$ is recommended for intrametatarsal pad injection in cats.

The optimal timing for TD enhancement ranged from 5 to 15 minutes in our study, similar to intrametatarsal pad injection in dogs and intrapopliteal lymph node injection in cats. ${ }^{20,22}$ In our experience, thoroughly massaging the pads and hind limbs increases the success rate and reduces the time required for TD enhancement. Massaging the injection site is also recommended for perianal subcutaneous injection of contrast medium. ${ }^{16,17}$ However, the relatively concentrated accumulation of contrast medium in the pads may result in pad and limb massage being more efficient and practical.

The combination of TD ligation, subtotal pericardiectomy, and cisterna chyli ablation or omentalization followed by placement of pleural ports has been the most common approach for treating cats with chylothorax. 14,15 Therefore, preoperative Iymphangiography is recommended for a complete understanding of the morphology of the TDs or cisterna chyli. ${ }^{30}$ Previous literature suggests a left-sided approach for TD ligation in cats and a right-sided approach in dogs with chylothorax.1,10,31 However, in our study, $72 \%$ of the TD branches were located in the right hemithorax, similar to that (78\% to $87 \%$ ) seen in dogs but inconsistent with previous reports of cats. ${ }^{17,20,21,28,32}$ Furthermore, most of the TDs at the T11 to T12 level had only 1 or 2 branches, making complete ligation at this site easier. Thus, a rightsided approach for TD ligation may also be considered in cats. In fact, the 3 cats that received surgery exhibited right or dorsal TD branches at the T10 to T12 level in the preoperative CT lymphangiography, and complete TD ligation was achieved by means of a right-sided approach. Our results revealed the potential variation of TD morphology in cats and highlighted the importance of preoperative CT Iymphangiography to guide the selection of approach side for TD ligation.

In our study, the cisterna chyli was located at the $L 2$ to $L 3$ level, medial to the middle of the right kidney and dorsal to the proximal descending colon. Compared with canine cisterna chyli, 20 which spans from the L2 to L5 level with the most common site being $L 3$, the location of feline cisterna chyli has relativeIy less variation among individuals. In contrast, the structure of cisterna chyli varies individually, since 5 types of cisterna chyli were identified in the small number of cases in our study. A left-sided 9th or 10th intercostal approach for cisterna chyli ablation has been described in cats with idiopathic chylothorax. ${ }^{14}$ However, 5 of 6 cats in our study exhibited right-sided distribution of the cisterna chyli, indicating that a right-sided approach may be considered if preoperative CT Iymphangiography is available. These results may be valuable to aid the selection of the best approach sites and corridor for cisterna chyli ablation, as suggested in a previous study of dogs. ${ }^{30}$

The TD failed to opacify in only 1 cat in the present study. A mass at the right third mammary gland with severe enlargement and heterogeneous enhancement of the bilateral internal iliac, medial iliac, and inguinal lymph nodes and blurring margin of the retroperitoneal fat were noted in this cat. These image findings suggested a mammary gland tumor with lymphatic metastasis or lymphoid tumors. However, further diagnostic workup or biopsy were declined by the owner and a definitive diagnosis was not available. Accumulation of the contrast medium was noted in the enlarged lymph nodes, and we presumed that the abnormal lymph nodes affected the lymphatic flow. However, successful migration of the contrast medium from the metatarsal region to the lymphatic vessels was still achieved.

Three systems of the superficial lymph vessels (superficial lateral, superficial medial, and deep medial systems) in the pelvic limbs have been described in dogs, and the identification of dorsal lymph vessel pathway, which courses along the gluteal region to the abdominal cavity, could be enhanced via intrametatarsal pad injection. ${ }^{20,33}$ Despite the fact that cats have similar lymphatic pathways, their anatomic structure in CT Iymphangiography has not been reported. ${ }^{34}$ The cats in the present study exhibited a higher frequency (66\%) of the dorsal lymph vessel pathway than dogs (34\%), possibly indicating a more complicated communication of superficial lymph vessels in cats. ${ }^{20}$ Inconsistent drainage pathway in different pelvic limbs was also noted in 3 cats in the present study, and a previous study demonstrated that this inconsistency might result in failure of CT lymphangiography via unilateral popliteal lymph node injection. ${ }^{22}$ Hence, bilateral intrametatarsal pad injection of contrast medium may cover most pathways of the hind limbs to increase the success rate of lymphatic vessel enhancement. Intrametatarsal pad injection may also be considered a possible method of staging the malignancy of pelvic limb disease.

The main limitations of this study were its retrospective design and small sample size. Multiple institutions were included in this study to increase the sample size; however, this resulted in different operators performing the procedures, which may have affected the results. Additionally, surgery was performed to confirm the number of TD branches in only 3 of 7 cases.

In conclusion, feline CT lymphangiography by intrametatarsal pad injection is a safe and effective method to enhance the TDs and cisterna chyli in cases of chylothorax. Future studies including a larger sam- 
ple size are warranted to establish the morphology of the feline TD and verify possible complications.

\section{Acknowledgments}

The authors received no financial support for the research, authorship, or publication of this article.

The authors declare no conflicts of interest with respect to the research, authorship, or publication of this article.

We thank Dr. Yi-Hsuan Lee for helping with data acquisition.

\section{References}

1. Fossum TW. Surgery of the lower respiratory system: pleural cavity and diaphragm. In: Small Animal Surgery. Elsevier; 2015:941-947.

2. Birchard SJ, Ware WA, Fossum TW, Fingland RB. Chylothorax associated with congestive cardiomyopathy in a cat. J Am Vet Med Assoc. 1986;189(11):1462-1464.

3. Donahoe JM, Kneller SK, Thompson PE. Chylothorax subsequent to infection of cats with Dirofilaria immitis. J Am Vet Med Assoc. 1974;164(11):1107-1110.

4. Forrester SD, Fossum TW, Rogers KS. Diagnosis and treatment of chylothorax associated with lymphoblastic Iymphosarcoma in four cats. J Am Vet Med Assoc. 1991;198(2):291-294.

5. Fossum TW, Forrester SD, Swenson CL, et al. Chylothorax in cats: 37 cases (1969-1989). J Am Vet Med Assoc. 1991;198(4):672-678

6. Fossum TW, Miller MW, Rogers KS, Bonagura JD, Meurs KM. Chylothorax associated with right-sided heart failure in five cats. J Am Vet Med Assoc. 1994;204(1):84-89.

7. Glaus TM, Jacobs GJ, Rawlings CA, Watson ED, Calvert CA. Surgical removal of heartworms from a cat with caval syndrome. J Am Vet Med Assoc. 1995;206(5):663-666.

8. Singh A, Brisson BA. Chylothorax associated with thrombosis of the cranial vena cava. Can Vet J. 2010;51(8):847-852.

9. McLane MJ, Buote NJ. Lung lobe torsion associated with chylothorax in a cat. J Feline Med Surg. 2011;13(2):135138.

10. Fossum TW, Mertens MM, Miller MW, et al. Thoracic duct ligation and pericardiectomy for treatment of idiopathic chylothorax. J Vet Intern Med. 2004;18(3):307-310.

11. Sicard GK, Waller KR, McAnulty JF. The effect of cisterna chyli ablation combined with thoracic duct ligation on abdominal lymphatic drainage. Vet Surg. 2005;34(1):64-70.

12. da Silva CA, Monnet E. Long-term outcome of dogs treated surgically for idiopathic chylothorax: 11 cases (19952009). J Am Vet Med Assoc. 2011;239(1):107-113.

13. Clendaniel DC, Weisse C, Culp WT, Berent A, Solomon JA. Salvage cisterna chyli and thoracic duct glue embolization in 2 dogs with recurrent idiopathic chylothorax. J Vet Intern Med. 2014;28(2):672-677.

14. Stockdale SL, Gazzola KM, Strouse JB, Stanley BJ, Hauptman JG, Mison MB. Comparison of thoracic duct ligation plus subphrenic pericardiectomy with or without cisterna chyli ablation for treatment of idiopathic chylothorax in cats. J Am Vet Med Assoc. 2018;252(8):976-981.

15. Reeves LA, Anderson KM, Luther JK, Torres BT. Treatment of idiopathic chylothorax in dogs and cats: a systematic review. Vet Surg. 2020;49(1):70-79.

16. Birchard SJ, McLoughlin MA, Smeak DD. Chylothorax in the dog and cat: a review. Lymphology. 1995;28(2):6472.

17. Kim M, Lee H, Lee N, et al. Ultrasound-guided mesenteric lymph node iohexol injection for thoracic duct computed tomographic lymphography in cats. Vet Radiol Ultrasound. 2011;52(3):302-305.

18. Esterline ML, Radlinsky MG, Biller DS, Mason DE, Roush JK, Cash WC. Comparison of radiographic and computed tomography lymphangiography for identification of the canine thoracic duct. Vet Radiol Ultrasound. 2005;46(5):391-395.

19. Johnson EG, Wisner ER, Kyles A, Koehler C, Marks SL. Computed tomographic lymphography of the thoracic duct by mesenteric lymph node injection. Vet Surg. 2009;38(3):361-367.

20. Lin LS, Chiu HC, Nishimura R, Fujiwara R, Chung CS. Computed tomographic lymphangiography via intra-metatarsal pad injection is feasible in dogs with chylothorax. Vet Radiol Ultrasound. 2020;61(4):435-443.

21. Millward IR, Kirberger RM, Thompson PN. Comparative popliteal and mesenteric computed tomography lymphangiography of the canine thoracic duct. Vet Radiol Ultrasound. 2011;52(3):295-301.

22. Dickerson VM, Grimes JA, Secrest SA, Wallace ML, Schmiedt CW. Abdominal lymphatic drainage after thoracic duct ligation and cisterna chyli ablation in clinically normal cats. Am J Vet Res. 2019;80(9):885-890.

23. Ando K, Kamijyou K, Hatinoda K, Shibata S, Shida T, Asari M. Computed tomography and radiographic lymphography of the thoracic duct by subcutaneous or submucosal injection. J Vet Med Sci. 2012;74(1):135-140.

24. Iwanaga T, Tokunaga S, Momoi Y. Thoracic duct lymphography by subcutaneous contrast agent injection in a dog with chylothorax. Open Vet J. 2016;6(3):238-241.

25. Kim K, Cheon S, Kang K, et al. Computed tomographic lymphangiography of the thoracic duct by subcutaneous iohexol injection into the metatarsal region. Vet Surg. 2020;49(1):180-186.

26. Kim K, Choen S, Hwang J, Jang M. CT Iymphangiography with contrast medium injection into the perianal subcutaneous region in a dog with chylothorax. J Vet Clin. 2018;35(6):299-301.

27. Mayer MN, Sweet KA, Patsikas MN, Sukut SL, Waldner CL. Frequency of an accessory popliteal efferent lymphatic pathway in dogs. Vet Radiol Ultrasound. 2018;59(3):365373.

28. Lee N, Won S, Choi M, et al. CT thoracic duct lymphography in cats by popliteal lymph node iohexol injection. Vet Radiol Ultrasound. 2012;53(2):174-180.

29. Naganobu K, Ohigashi Y, Akiyoshi T, Hagio M, Miyamoto T, Yamaguchi R. Lymphography of the thoracic duct by percutaneous injection of iohexol into the popliteal lymph node of dogs: experimental study and clinical application. Vet Surg. 2006;35(4):377-381.

30. Morris KP, Singh A, Holt DE, et al. Hybrid single-port laparoscopic cisterna chyli ablation for the adjunct treatment of chylothorax disease in dogs. Vet Surg. 2019;48(S1):0121-0129. doi:10.1111/vsu.13195

31. Fossum TW. Chylothorax in cats: is there a role for surgery? J Feline Med Surg. 2001;3(2):73-79.

32. Lindsay FE. Chylothorax in the domestic cat-a review. J Small Anim Pract. 1974;15(4):241-258.

33. Evans HE. The lymphatic system. In: Miller's Anatomy of the Dog. 4th ed. Elsevier; 2013:555-556.

34. Ratzlaff MH. The superficial lymphatic system of the cat. Lymphology. 1970;3(4):151-159.

\section{Supplementary Materials}

Supplementary materials are posted online at the journal website: avmajournals.avma.org 